\title{
Shiftwork, functional bowel symptoms, and the microbiome
}

\author{
Ann E. Rogers ${ }^{\text {Corresp., } 1}$, Yi-Juan Hu ${ }^{2}$, Ye Yue ${ }^{2}$, Emily F. Wissel ${ }^{1}$, Robert A. Petit III ${ }^{3}$, Simone Jarrett ${ }^{4}$, Jennifer \\ Christie $^{5}$, Timothy D. Read ${ }^{6}$ \\ 1 \\ ${ }^{1}$ Nell Hodgson Woodruff School of Nursing, Emory University, Atlanta, Georgia, United States \\ Department of Biostatistics and Bioinformatics, Rollins School of Public Health, Emory University, Atlanta, Georgia, United States \\ 3 Investigational Clinical Microbiology Core, Emory University, Atlanta, Georgia, United States \\ 4 Einstein Medical Center Philadelphia, Philadelphia, Pennsylvania, United States \\ 5 Division of Digestive Diseases, Emory School of Medicine, Emory University, Atlanta, Georgia, United States \\ 6 Division of Infectious Diseases, Emory School of Medicine, Emory University, Atlanta, Georgia, United States \\ Corresponding Author: Ann E. Rogers \\ Email address: ann.e.rogers@emory.edu
}

Background. There are about 15 million Americans working full-time on evening, night, or rotating shifts. Between $48 \%$ and $81.9 \%$ of those working rotating or night shifts report abdominal pain, constipation, diarrhea and other symptoms of functional bowel disorders. The basis for this high prevalence of functional bowel disorders, including irritable bowel syndrome (IBS), among shift workers is unknown. Animal studies, however, suggest that circadian disruption, similar to that in shift workers, may contribute to the development of $\mathrm{Gl}$ complaints among shift workers by altering the composition and normal diurnal rhythmicity of the resident intestinal microbes. Therefore, the present study was designed to determine if there were differences in 1) composition and diversity of the microbiome of night shift workers compared to day shift workers; and 2) the composition and diversity of the microbiome among shift workers experiencing functional bowel symptoms compared to shift workers who did not experience functional bowel symptoms.

Methods. Fifty-one full time staff nurses who worked either 12-hour day or night shifts completed demographic information, and the Rome III IBS module. They also collected two samples of gut microbiota before the beginning and at the end of their last work shift on day 14, using validated fieldtested methods consistent with the Human Microbiome Project. After DNA extraction, 16S rRNA sequencing and assignment to the genus level was completed, samples were then compared to determine if there were 1 ) differences in the diversity and profile of the microbiome by shift type; 2 ) if there were differences in the microbiome by time of day for collection; and 3) whether there were differences in the diversity and profile of the microbiome of nurses with IBS and those without IBS. Results. There were no differences in alpha or beta diversity of gut microbiota when specimens from day and night shift nurses were compared. There were however marginal differences in beta diversity when specimens collected at the beginning and end of the shifts were compared, with seven OTUs being differentially abundant when collected from day shift workers in the evening. There were also three OTUs to be differentially abundant in participants reporting IBS symptoms. 


\section{Shiftwork, Functional Bowel Symptoms and the}

\section{Microbiome}

3

Ann E. Rogers ${ }^{1}$, Yi-Juan $\mathrm{Hu}^{2}$, Ye Yue ${ }^{3}$, Emily F. Wissel ${ }^{4}$, Robert A. Petit III ${ }^{5}$, Simone Jarrett ${ }^{6}$, Jennifer Christie 7 , Timothy D. Read ${ }^{8}$

${ }^{1}$ Nell Hodgson Woodruff School of Nursing, Emory University, Atlanta, Georgia, USA

2 Department of Biostatistics and Bioinformatics, Rollins School of Public Health, Emory University, Atlanta, Georgia, USA

${ }^{3}$ Department of Biostatistics and Bioinformatics, Rollins School of Public Health, Emory University, Atlanta, Georgia,

${ }^{4}$ Nell Hodgson Woodruff School of Nursing, Emory University, Atlanta, Georgia, USA ${ }^{5}$ Investigational Clinical Microbiology Core, Emory University, Atlanta, Georgia, USA

${ }^{6}$ Einstein Medical Center Philadelphia, Pennsylvania, USA

${ }^{7}$ Division of Digestive Diseases, Emory School of Medicine, Emory University, Atlanta, Georgia, USA

${ }^{8}$ Division of Infectious Diseases, School of Medicine, Emory University, Atlanta, Georgia, USA

Ann E. Rogers ${ }^{1}$

1520 Clifton Road NE, Atlanta, Georgia, 30322, USA

Email address: ann.e.rogers@emory.edu

Corresponding Author:

\section{Abstract}

Background. There are about 15 million Americans working full-time on evening, night, or rotating shifts. Between $48 \%$ and $82 \%$ of those working rotating or night shifts report abdominal pain, constipation, diarrhea and other symptoms of functional bowel disorders. The basis for this high prevalence of functional bowel disorders, including irritable bowel syndrome (IBS), among shift workers is unknown. Animal studies, however, suggest that circadian disruption, similar to that in shift workers, may contribute to the development of gastrointestinal complaints among shift workers by altering the composition and normal diurnal rhythmicity of the resident intestinal 
34 microbes. Therefore, the present study was designed to determine if there are

35 differences in 1) composition and diversity of the microbiome of night shift workers

compared to day shift workers; and 2) the composition and diversity of microbiome among shift workers experiencing functional bowel symptoms compared to shift workers not experiencing functional bowel symptoms.

Methods. Fifty-one full time staff nurses who worked either 12-hour day or night shifts completed demographic information, and the Rome III IBS module. They also collected two samples of fecal microbiota before the beginning and at the end of their last work shift on day 14, using validated field-tested methods consistent with the Human Microbiome Project. After DNA extraction, 16S rRNA sequencing and assignment to the genus level was completed, samples were then compared to determine if there were 1) differences in the diversity and profile of the microbiome by shift type; 2 ) if there were differences in the microbiome by time of day for collection; and 3) whether there were differences in the diversity and profile of the microbiome of nurses with IBS and those without IBS.

Results. There were no differences in alpha or beta diversity of fecal microbiota when specimens from day and night shift nurses were compared. There were however marginal differences in beta diversity when specimens collected at the beginning and end of the shifts were compared, with seven Amplicon Sequence Variant (ASVs) being differentially abundant when collected from day shift workers in the evening. There were also three ASVs to be differentially abundant in participants reporting IBS symptoms. 
57

58

59

60

61

62

63

64

65

66

67

68

69

\section{Introduction}

There are about 15 million Americans working full-time on evening, night, or rotating shifts, or other irregular employer-arranged schedules; $4.7 \%$ on evening shifts, $3.2 \%$ on night shifts, $3.1 \%$ on irregular schedules, and $2.5 \%$ on rotating shifts (United States Department of Labor Bureau of Labor Statistics 2005). Night shift work is associated with increased mortality, higher risk of cardiovascular disease, cancer, diabetes, - hypertension, chronic fatigue, sleep problems and higher body weight (Gu 2015; Jia 2013; Myers 2015; Pan 2011; Rajaratnam 2011; Vyas 2012). Night and rotating shift workers also report a higher prevalence of Irritable Bowel Syndrome (IBS), abdominal pain, constipation and diarrhea than do day shift workers (Caruso 2004; Knutsson 2010; Nojkov 2010; Wells 2012). In fact, between $48 \%$ and $82 \%$ of those working rotating or night shifts report abdominal pain, constipation, diarrhea and other symptoms of functional bowel disorders (Nojkov 2010; Saberi 2010).

The basis for this high prevalence of functional bowel disorders, including IBS, among shift workers is unknown. However, some studies suggest that inappropriate nutrition or irregularity in the timing of meals (Bilski 2006; Lowen 2010), and psychological disorders (Zhen 2006) may contribute to the high prevalence of functional bowel symptoms among workers on rotating or night shifts.

Other studies strongly suggest that sleep deprivation or sleep disturbances are associated with the presence and severity of functional bowel symptoms reported by resident physicians and nurses (Jarrett 2000; Saberi 2010; Wells 2012). Moreover, 
82 animal studies suggest that circadian disruption, similar to that in shift workers,

83 contributes to the development of gastrointestinal (GI) complaints among shift workers

84 by altering the composition and normal diurnal rhythmicity of the resident intestinal

85 microbes (De Bacquer 2009). Interestingly, gut microbiota community composition and

86 diversity are malleable and sensitive to changes in diet and other environmental factors

87 (Voigt 2014), including activity and sleep patterns. For example, Thaiss and colleagues

88 (Thaiss 2014) "jet lagged" a group of mice by subjecting them to an 8-hour advance for

89 three days before allowing them to revert to their usual schedule for three more days,

90 then subjecting them to another 8 hour advance for three days. Mice exposed to 4

91 weeks of this schedule lost their usual pattern of physical activity, and consumed food at

92 irregular intervals. Significantly, this environmentally induced disruption of daily activity

93 patterns (jet lag schedule) was associated with a loss of diurnal rhythmicity of

94 microbiota composition in mice.

95

96 Thaiss and colleagues (Thaiss 2014) also found similar changes in human microbiota

97 composition in two volunteers who flew from the US to Israel (an 8-10 hour advance).

98 Samples collected at baseline (one day pre-flight), during jet lag (one day after landing),

99 and during recovery (2 weeks after landing) showed rapid changes in the composition of

100 the microbiota. During jet lag (the first 24-hours after landing), there was a higher

101 relative representation of Firmicutes, which reversed upon recovery from jet lag (2

102 weeks later). It is unknown it these changes would occur with the chronic circadian

103 disruption experienced by night shift workers. Although some studies have

104 demonstrated no differences in composition of the fecal microbiome when samples from 
105 lean and obese individuals were compared (Ley 2006; Turnbaugh 2009), other studies

106 in humans have demonstrated that Firmicutes are associated with a higher propensity

107 for obesity and metabolic disease (Finucane 2014; Ley 2006), conditions that are more

108 common in night and rotating shift workers (De Bacquer 2009; Suwazono 2008).

109

110 Finally, multiple studies have linked reduced microbial diversity and richness in

111 microbial communities to IBS symptoms. For example, Krogius-Kurikka and colleagues

112 (Krogius-Kurikka 2009) reported that fecal samples from patients with diarrhea-

113 predominant IBS were enriched with Proteobacteria and Firmicutes but had reduced

114 Actinobacteria and Bacteroidetes compared to healthy controls. Other studies (Bhattarai

115 Y. 2017; Salonen 2010) have shown an increase in the Firmicutes-to-Bacteriodetes-

116 ratio, a decrease in some types of Firmicutes families (Lactobacilli, Faecalibacterium)

117 and the Actinobacteria population (Bifidobacteria, Collinsella), and an increase in some

118 Firmicutes families (Veillonella, Streptococci, and Ruminococcus spp.) and in

119 Proteobacteria (Enterobacteriaceae spp.). In addition, low microbial richness, an

120 absence of Methanobacteriales, and enrichment with Bacteroides enterotypes are

121 associated with more severe IBS symptoms (Tap 2017). Not only is the composition of

122 the fecal microbiota altered in patients with IBS, these imbalances in the microbial

123 community or dysbiosis, occur more frequently in patients with IBS compared to healthy

124 individuals. Reduced diversity was observed in nearly three-fourths of the IBS patients

125 studied by Casén and colleagues (Casén 2015) compared to 16\% in normal individuals

126 (Collins 2014; Jeffery 2012). 
128 While these studies suggest that shiftwork alters gut microbiota and that alterations in

129 gut microbiota are common in patients with IBS, they do not demonstrate whether these

130 alterations are associated with the somatic symptoms experienced by many rotating

131 and night shift workers. Therefore, the present study is designed as the initial step in

132 determining if there are differences in 1) composition and diversity of the microbiome of

133 night shift workers compared to day shift workers; and 2) the composition and diversity

134 of microbiome among night shift workers experiencing functional bowel symptoms (e.g.,

135 bloating, lower abdominal pain, constipation and diarrhea) compared to night shift

136 workers not experiencing functional bowel symptoms.

137

138

139

140

141

142

143

144

145

146

147

148

149

150

151

152

\section{Materials \& Methods}

\section{Subjects}

Participants in this study included 51 full-time staff nurses who worked 12-hour day or night shifts at a large university hospital. Registered nurses were eligible to participate if they were between the ages of 18 and 65 and did not report a history of inflammatory bowel disease (e.g., Crohn's disease or ulcerative colitis) or other chronic disorder affecting the GI track (e.g., GI cancer). Those with recent antibiotic exposure were asked to delay their enrollment in the study for two weeks after their last dose of antibiotics.

As expected, the sample was predominantly female (96\%), with a mean age of $32.9 \pm$ 10.0 years and a range of 21-59 years. Just under half of the participants reported working straight day shifts (47\%), with the remainder of the sample working either straight night shifts $(51 \%)$ or rotating shifts $(2 \%)$. For purposes of the analysis, the nurse 
153 who reported working rotating shifts was categorized as working night shift since she

154 worked straight nights during the two-week data-gathering period. Although only three

155 participants (6\%) reported a prior diagnosis of IBS, a total 18 participants (35\%) met

156 criteria for the diagnosis of IBS using the Rome III criteria. Participant BMIs ranged from

15718.2 to 39.5 with a mean BMI of $26.7 \pm 5.4$. As illustrated in Table 1, there were no

158 significant differences by shift type in terms of age, BMI, diagnosis of IBS or type of IBS.

159

160

Instruments

161 Data for this pilot study was obtained using a variety of subjective and objective

162 measures. A Demographic Questionnaire and Brief Health History was used to collect

163 information about participant age, and the usual shift worked. Participants were also

164 asked to report any previous diagnosis of inflammatory bowel disorders or chronic

165 diseases affecting the $\mathrm{GI}$, and to list current medications and supplements used. The

166 IBS module from the Rome III Questionnaire (2006) consists of 10 questions that ask

167 subjects to rate the frequency of recurrent abdominal pain or discomfort, onset of pain

168 associated with a change in frequency of stools, and the onset of pain associated with a

169 change in the form of stools. This module is considered the gold standard for assessing

170 functional bowel symptoms.

172 Samples of fecal microbiota were collected just before the beginning and just after the

173 end participants' work shift at the end of the two week data-collection period using

174 validated, field-tested methods consistent with the Human Microbiome Project (Methé

175 2012). Four specimens (two each time) were collected using the Elution-swap system

176 (Copan). The rectal swabs were stored in $1 \mathrm{~mL}$ of Amies transport medium (Copan) and 
177 immediately frozen and stored until DNA extraction. Prior to extraction, fecal material

178 (200 mg) was suspended in 500- $\mu$ lysozyme $(20 \mathrm{mg} / \mathrm{ml}$ in $20 \mathrm{mM}$ Tris-HCl pH 8, $2 \mathrm{mM}$

179 EDTA, $1.2 \% \mathrm{w} / \mathrm{v}$ Triton $\mathrm{X}-100$ ) and incubated at $37^{\circ} \mathrm{C}$ for $2 \mathrm{~h}$ using the QIAamp ${ }^{\circledR}$ DNA

180 Stool Mini Kit (Qiagen, Inc., Valencia, CA).

181

182 Procedure

183 After obtaining approval from the Emory University's IRB (MOD001-IRB00089064) and

184 Emory Healthcare's Nursing Research Council, emails describing the study were sent to

185 all staff nurses. Those interested in participating were instructed to contact the research

186 team to schedule an appointment to provide informed consent, review study

187 procedures, and complete the demographic and Rome III questionnaire. After written

188 informed consent was obtained, the participant's work schedule was then reviewed to

189 determine an appropriate date to collect samples of fecal microbiota at the beginning

190 and end of the participant's shift.

191

192 Data Analysis

193 DNA extraction \& 16S rRNA sequencing_DNA extraction and 16S sequencing was

194 performed by Omega Bioservices (Norcross, GA, USA) using a standard protocol. DNA 195 was isolated using Omega Biotek Mag-Bind® Universal Pathogen DNA Kit. The V3-V4 region of the bacterial $16 \mathrm{~S}$ rRNA gene sequences were amplified using the primer pair containing the gene-specific sequences and Illumina adapter overhang nucleotide sequences. The full length primer sequences are: 16S Amplicon PCR Forward Primer

199 (5'- TCGTCGGCAGCGTCAGATGTGTATAAGAGACAGCCTACGGGNGGCWGCAG) 
200 and 16 S Amplicon PCR Reverse Primer (5'-

201 GTCTCGTGGGCTCGGAGATGTGTATAAGAGACAGGACTACHVGGGTATCTAATCC)

202 . For amplicon PCR, each $25 \mu \mathrm{L}$ of polymerase chain reaction (PCR) reaction contained

$20312.5 \mathrm{ng}$ of sample DNA as input, $12.5 \mu \mathrm{L} 2 \mathrm{x}$ KAPA HiFi HotStart ReadyMix (Kapa

204 Biosystems, Wilmington, MA) and $5 \mu \mathrm{L}$ of $1 \mu \mathrm{M}$ of each primer. PCR reactions were

205 carried with an initial denaturation step performed at $95^{\circ} \mathrm{C}$ for $3 \mathrm{~min}$ followed by 25

206 cycles of denaturation $\left(95^{\circ} \mathrm{C}, 30 \mathrm{~s}\right)$, annealing $\left(55^{\circ} \mathrm{C}, 30 \mathrm{~s}\right)$ and extension $\left(72^{\circ} \mathrm{C}, 30\right.$

$207 \mathrm{sec}$ ), and a final elongation of $5 \mathrm{~min}$ at $72^{\circ} \mathrm{C}$. PCR product was cleaned up from the 208 reaction mix with Mag-Bind RxnPure Plus magnetic beads (Omega Bio-tek, Norcross,

209 GA). A second index PCR amplification, used to incorporate barcodes and sequencing 210 adapters into the final PCR product, was performed in $25 \mu \mathrm{L}$ reactions, using the same

211 master mix conditions as described above. Cycling conditions were as follows: $95^{\circ} \mathrm{C}$ for 2123 minutes, followed by 8 cycles of $95^{\circ} \mathrm{C}$ for $30^{\prime \prime}, 55^{\circ} \mathrm{C}$ for $30^{\prime \prime}$ and $72^{\circ} \mathrm{C}$ for $30^{\prime \prime}$. A final, 5 213 minutes' elongation step was performed at $72^{\circ} \mathrm{C}$. The libraries were normalized with 214 Mag-Bind® EquiPure Library Normalization Kit ((Omega Bio-tek, Norcross, GA) then 215 pooled. The pooled library $\sim 600$ bases in size was checked using an Agilent 2200 216 TapeStation and sequenced $(2 \times 300$ bp paired-end read setting) on the MiSeq 217 (Illumina, San Diego, CA). Sequence data was submitted to the National Center for 218 Bioinformatic Information Short Read Archive database: accession PRJNA687007.

Processing of sequence data and taxonomic assignment to Amplicon Sequence

221 Variants (ASVs). Data processing, including demultiplexing, QC filtering,

222 contamination and sample mislabeling data checks, ASV representation, taxonomy 
223 assignment via a reference database (Caporaso 2010; Wang 2007), and phylogeny and 224 diversity analysis (Lozupone 2007; Lozupone 2006; Lozupone 2005) was done using R 225 packages dada2 and phyloseq (Callahan 2016; McMurdie 2013). After filtering for low 226 quality reads and filtering possible contaminants, we obtained a range of $12,455-$ 119,605 reads per sample with a mean of $33,933(S D=10,412$ and a median of 33,452 228 (scripts used to generate this data have been included as supplemental files dada2229 analysis 1 , and dada2-analysis 2 )

\section{Statistical Analysis}

232

All statistical analyses were conducted using R package 3.6.3. A p-value less than 0.05 233 was considered statistically significant.

We computed the Chao1 (measuring species richness) and Shannon (measuring both 236 richness and evenness) alpha diversity indices for each sample, since loss of taxonomic diversity in general is an indicator of disease state in many ecological systems. We log238 transform Chao1 to be at the same scale as Shannon. We also computed the BrayCurtis (measuring dissimilarity/distance of each pair of samples based on relative abundance data) and Jaccard (based on presence-absence data) beta diversity distance metrics. We used Principal Coordinates Analysis (PCoA) to visualize clustering 242 of the samples based on these distance metrics.

244 Given that this is a pilot study with 51 subjects, the primary focus was analyzing the 245 microbiome profiles (in terms of alpha diversity, beta diversity, and individual genera at 
246 the relative abundance and presence-absence scales) for (a) differences between day 247 and night shift workers, (b) differences between the beginning and the end of the shift 248 (main effect of time) and whether the differences depend on the shift type (time and shift 249 type interaction), and (c) differences between subjects with and without IBS symptoms. 250 Since each subject is measured four times (two at the beginning of the shift and two at 251 the end) for the microbiome, this study has a matched-set design. Care should be taken 252 in analyzing such matched-set data because the samples from the same subjects tend 253 to have positive correlations and the samples taken at the same time for the same 254 subjects tend to have even stronger positive correlations. While the analyses in (a) and 255 (c) consist of two-group comparisons "between" sets, the analyses in (b) consist of two256 group comparison "within" sets for the main effect of time as well as two-group 257 comparison of the change (from the beginning to the end of the shift) "between" sets. We have developed the Linear Decomposition Model (LDM) method and extended the existing PERMANOVA method that both can handle all of these analyses [Hu and Satten 2020; Zhu et al., in press]. While PERMANOVA performs comparison at the community level based on a beta-diversity distance matrix, the LDM performs the same 262 community-level comparison based on the relative abundance or presence-absence data. In addition, the LDM performs comparison at individual-genus level (e.g., detecting 264 individual genera that are differently "abundant" or "present" between groups) with falsediscovery rate control at the nominal level $10 \%$. We also use the LDM for comparing the 266 alpha diversity by treating it as a single genus.

\section{Results}


270 The PCoA plot in Figure 1 shows that samples obtained from the same participants tend

271 to cluster together and samples from the same time on sample participants tend to

272 cluster even more closely. This observation is consistent with the notion that

273 microbiome samples are homogeneous within subjects and heterogenous across

274 subjects, and thus confirms good quality of our data. Supplemental text (phyloseq

275 analysis) contains plots showing the composition of the samples at taxonomic different

276 levels

277

278 As shown in Figure 2, there were no significant differences in Chao1 $(p=0.411$ by the

279 LDM) and Shannon $(p=0.242)$ between day and night shift nurses. Nor were there 280 differences in Bray-Curtis ( $p=0.476$ by PERMANOVA) and Jaccard distances $(p=0.625)$

281 by shift type (Figure 3 ). Nor were there differences in relative abundance ( $p=0.489$ by

282 the LDM $)$ and presence-absence $(p=0.824)$ data across all genera.

283

284 Figure 4 shows an increase of the log Chao1 index from the beginning to the end of the 285 shift for day-shift workers while a decrease for night-shift workers, and this difference of 286 change is significant (interaction $p=0.034$ by the LDM); so does the Shannon index $287(p=0.008)$. However, the change among the pooled workers was not significantly 288 different (main effect of time $p=0.473$ for Chao 1 and $p=0.236$ for Shannon) possibly due 289 to the cancellation of effects with opposite directions. In terms of beta diversity, the 290 change of the beta diversity metric from the beginning to the end of the shift among the 291 pooled workers was marginally significant or significant (main effect of time $p=0.056$ by 292 PERMANOVA for Bray-Curtis and 0.014 for Jaccard). Marginally significant and 
293 significant findings were also noted by the LDM (main effect of time $p=0.035$ and 0.063 294 based on relative abundance and presence-absence data, respectively). However, 295 there is not enough evidence to confirm that the change was significantly different 296 between day shift and night shift workers possibly due to the small sample size, 297 although there was suggestive evidence (interaction $p=0.192$ and 0.118 by 298 PERMANOVA based on Bray-Curtis and Jaccard, respectively; $p=0.320$ and 0.134 by 299 the LDM based on the relative abundance and presence-absence data). In addition, the 300 LDM revealed seven ASVs to be differentially abundant between the beginning and the 301 end of the shift; ASV_455(S5-A14a, more abundant at the beginning), 302 ASV_2527(Ruminoccaceae_NK4A214_group, more abundant at the end), ASV_1304(Ruminococcus_1, more abundant at the end), ASV_221 (Mobiluncus, more 304 abundant at the beginning), ASV_7(Campylobacter), ASV_130(Alistipes, more 305 abundant at the end), and ASV_62(Agathobacter, more abundant at the end). 306

307 Finally, as shown in Figure 5 there were no significant difference in alpha diversity when 308 comparing participants without and with IBS symptoms $(p=0.849$ for Chao $1, p=0.484$ 309 for Shannon, by the LDM method). Although there were no differences in beta diversity 310 ( $p=0.206$ for Bray-Curtis and $p=0.213$ for Jaccard) by whether or not the participant had 311 symptoms of IBS), there were some significant differences based on the LDM results. 312 Specifically, three ASVs, ASV_1160 (Flavonifractor), ASV_1134 (Oscillibacter) and 313 ASV_2379 (Ruminiclostridium_9), were detected to be differentially abundant. Three 314 ASVs, ASV_1160 (Flavonifractor), ASV_2379 (Ruminiclostridium_9) and ASV_47 
315 (Escherichia/Shigella), were detected to be significantly more likely to be present in 316 participants reporting IBS symptoms.

317

318

319

320

321

322

323

324

325

326

327

328

329

330

331

332

333

334

\section{Discussion}

The findings of this pilot study suggest that there are no differences in the richness and diversity of species when samples from nurses working day and night shifts were compared. There were however some statistically significant changes in both alpha and beta diversity metrics when specimens collected at the beginning and end of the shifts were compared and there was also some evidence that the changes were different for day shift and night shift workers, with increased alpha diversity noted at the end of the day shift and decreased alpha diversity noted at the end of the night shift. Seven ASVs were found to be differentially abundant between the beginning and end of the shifts for the entire sample. In addition, there were three ASVs to be differentially abundant in participants reporting IBS symptoms.

Studies comparing the effects of shift work on the gut microbiome are limited and somewhat contradictory. For example, a study of 10 male security guards who worked both day and night shifts found there were no significant differences in in alpha or beta diversity within and across-subject variation for both shifts (Mortas 2020). In contrast, slight changes in microbial abundance and diversity were noted when 22 subjects, aged 20-35 years, delayed their sleep period for 2-4 hours (Liu 2020). Although there have been studies comparing circadian variation in the gut microbiota in mice (Thaiss 2014) and another describing the results samples collected during multiple time points over several days by two subjects (Thaiss 2014), our study is the first to compare the 
340 richness and diversity of gut microbiota collected from 51 human participants at two

341 different time points in 24 hours.

342

343 Even though there are numerous studies that have reported increased and/or

344 decreased amounts of various gut bacteria among patients with IBS (Bhattarai Y. 2017;

345 Casén 2015; Pittayanon 2019; Salonen 2010; Tap 2017), a recent systematic review

346 found only nine studies that discussed differences in alpha-diversity in patients with IBS

347 compared to normal controls (Pittayanon 2019). Slightly over half of the studies $(55.6 \%)$

348 reported a significant decrease in the richness and diversity in patients with IBS (Carroll

349 2011; Carroll 2012; Liu 2016; Rangel 2015), whereas the remaining four studies (Carroll

350 2012; Durban 2012; Rigsbee 2012; Tap 2017), like our current study, revealed no

351 differences in alpha-diversity compared to healthy controls. A more elaborate study

352 (Pozuelo 2015) comparing fecal and mucosa-associated microbiome samples obtained

353 from the sigmoid colon during colonoscopy, not only found no differences in alpha

354 diversity when the two types of samples were compared, but that there were no

355 differences in richness and diversity when samples obtained from healthy controls and 356 patients with IBS or IBS subtypes were compared.

358 Like other studies comparing patients with IBS symptoms to healthy controls, our pilot

359 study found increased Firmicutes (Chong 2019), specifically Flavonifractor,

360 Oscillibacter, and Ruminiclostudium among participants with IBS (Casén 2015). The

361 increased abundance of E. coli/ Shigella possibly reflects the suspected relationship

362 between Shigellosis and IBS (Youn 2016). In contrast, Pozuelo et al (Pozuelo 2015) 
363 reported that Methanobacteriaceae, Ruminococcaceae, Erysipleotrichacea, and one

364 unknown Clostridiales family were all found to be decreased in patients with IBS-M

365 (IBS-mixed type) and IBS-D (IBS-diarrhea), but not in patients with IBS-C (IBS-

366 constipation).

367

368 IBS is estimated to have a world-wide prevalence of 10-15\% (Canavan 2014; Sperber 369 2017). Prevalence rates of IBS are typically higher among nurses, with rates ranging from $17.4 \%$ in China (Liu 2014) to $45.2 \%$ in Nigeria (Akere 2014). The prevalence rate 371 of IBS among study participants was $35 \%$, quite similar to the prevalence rate reported among nurses at the University of Michigan Medical Center (36.6\%) (Nojkov et al. 373 2010). However, the study of hospital staff nurses (Nojkov et al. 2010), and other 374 studies of shift workers (Kim 2013), there were no differences in the prevalence of IBS symptoms among day and night shift nurses in our study.

376

This study is limited by a number of factors. First, our study population consisted of a 378 convenience sample of nurses that might not be representative of the larger nursing workforce or the larger population of shift workers. The overall participation rate was relatively low, which raises concerns about how representative the participants were of the total population of nurses who were invited to participate. Additionally, given the

382 focus of the study, nurses who experienced IBS symptoms may have been more likely 383 to participate than those who did not experience IBS symptoms. Finally, the severity of 384 IBS symptoms and quality of life was not assessed, two factors which may have been 385 impacted by gut microbiome diversity and richness. 


\section{Conclusions}

388

389 There were no statistically significant differences in the richness and diversity when

390 samples of the gut microbiome from nurses working day and night shifts were

391 compared. However, when specimens collected at the beginning and ends of the shifts

392 were compared, there were some statistically significant or marginally differences in

393 alpha and beta diversity. Three ASVs were more common in participants reporting IBS

394 symptoms.

395 
396

397

398

399

400

401

402

403

404

405

406

407

408

409

410

411

412

413

414

415

416

417

418

419

420

421

422

423

424

425

426

427

428

429

430

431

432

433

434

435

436

437

438

439

440

\section{References}

2006. The Rome III Adult Criteria for Functional Gastrointestinal Disorders. McLean, VA: Degnon Associates.

Akere A, Akande, K.O. 2014. Association between Irritable Bowel Syndrome and Shift Work: Prevalence and Associated Factors among Nurses. Journal of Gastroenterology and Hepatology Research 3:1328-1331.

Bhattarai Y. M, Pedrogo, D.A., Kashyap, P.C. 2017. Irritable bowel syndrome: A gut microbiota-related disorder. American Journal of Physiology: Gastrointestiinal and Liver Physiology 312:52-62. 10.1152/ajpgi.0038.2016

Bilski B. 2006. Influence of shift work on diet and gastrointestional complaints among nurses: A pilot study. Medical Practice 57:15-19.

Callahan BJ, McMurdie, P.J., Rosen, M.J., Han, A.W., Johnson, A.J.A., Holmes, S.P. 2016. DADA2: High-resolution sample inference from Illumnia amplicon data. Nature Methods 13:581-183. 10.1038/nmeth.3869

Canavan C, West, J., Card, T. 2014. The epidemiology of irritable bowel syndrome. Clinical Epidemiology 6:71-80. 10.2147/CLEP.S40245

Caporaso JG, Bittinger, K., Bushman, F.D., DeSantis, T.Z., Anderson, G.L., Knight, R. 2010. PyNAST: a flexible tool for aligning sequences to a template alignment. Bioinformatics 26:266-267.

Carroll IM, Ringel-Kulka, T, Keku, T.,O, Chang, Y-H, Packey, C.D., Sarto, R.B., Ringel, Y. 2011. Molecular analysis of the luminal-and muscosal-associated intestinal microbiota in patients with diarrhea-predominant irritable bowel syndrome. American Journal of Physiology, Gastrointestinal and Liver Physiology 301:G799-G807.

Carroll IM, Ringel-Kulka, T, Siddle, J.P., Ringel, Y. 2012. Alterations in the composition and diversity of the intestional microbiota in patients with diarrhea-predominant irritable bowel syndrome. Neurogastroenterology Motility 24:521-530.

Caruso C, Lusk, S., Gillespie, B. 2004. Relationship of work schedules to gastroinstestional diagnosis, symptoms, and medication use in auto factory workers. American Journal of Internal Medicine 46:586-598.

Casén C, Vebø, H.C., Sekelja, ., Hegge, F.T., Kalsson, M.K., Ciemniejewska, E., Dzankovic, S., Frøyland, C., Nestestog, R., Engstarand, L., Munkholm, P., Nielsen, O.H., Rogler, G., Simrén, Öhman, L., Vatn, M.H., Rudi, K. 2015. Deviations in human gut microbiota: A novel diagnostic test for determining dysbiosis in patients with IBS or IBD. Alimentary Pharmacology and Theraputics 42:71-83.

Chong PPC, V.K. Looi, C.Y., Wong, W.F., Madhavan, P., Yong, V.C. 2019. The Microbiome and Irritable Bowel Syndrome-A Review on the Pathophysiology, Current Research, and Future Therapy. Frontiers in Microbiology 10:1-23. 10.3389/fmicb.2019/01136

Collins SM. 2014. A role for the gut microbiota in IBS. Nature Reviews Gastroenterology \& Hepatology 11:497-505. 10.1038/nrgastro.2014.40

De Bacquer D, van Risseghem, M., Clays, E., Kittel, F., De Backer, G., Braeckman, L. 2009. Rotating shift work and the metabolic syndrome: A prospective study. International Journal of Epidemiology 38:848-854.

Durban A, Abellan, J.J., Jimenez-Hernandez, N., Salgardo, P., Ponce, M., Ponce, J., Garrigues, V., Latorre, A., Moya, A. 2012. Structural laterations of faecal and mucosa-associated 
441

442

443

444

445

446

447

448

449

450

451

452

453

454

455

456

457

458

459

460

461

462

463

464

465

466

467

468

469

470

471

472

473

474

475

476

477

478

479

480

481

482

483

484

485 communities in irritable bowel syndrome. Enviromental Microbiology Reports 4:242247.

Finucane MM, Sharpton, T.J., Laurent, T.J., Pollard, K.S. 2014. A taxonomic signature of obesity in the microbiome? Getting to the guts of the matter. PLOS One 9:e84689.

Gu F, Han, J., Laden, F., Pan, A., Caporaso, N.E., Stampfer, M.J., Kawachi, I., Rexrode, K.M., Willett, W.C., Hankinson, S.E., Speizer, F.E., Schernhammer, E.S. 2015. Total and Cause-Specific Mortality of U.S., Nurses Working Rotating Night Shifts. American Journal of Preventive Medicine 48:241-252.

Jarrett M, Heitkemper, M., Cain, K.C., Burr, R.I., Hertig, V. 2000. Sleep disturbance influences gastrointestonal symptoms in women with irritable bowel syndrome. Digestive Diseases and Sciences 45:952-959.

Jeffery JB, O’Toole, P.W., Öhman, L., Claesson, M., Deane, J., Quigley, E.M.M., Simrén, M. 2012. An irritable blowel syndrome subtype defined by species-specific alterations in faecal microbiota. Gut Pathology 61:997-1006. 10.1136/gut/nl-2011-301501

Jia Y, Lu, Y., Wu, K., Lin, Q., Wei, S., Zhu, M., Huang, S., Chen, J. 2013. Does night work increase the risk of breast cancer? A systematic review and meta-analysis of epidemiological studies. Cancer Epidemiology 37:197-206.

Kim HI, Choi, J.Y., Kim, S-E., Jung, H-K., Shim, K-N, Yoo, K. 2013. Impact of shiftwork on irritable bowel sydrome and functional dyspepsia. Journal of Korean Medical Sciences 28:431-437. 10.3346/jkms.2013.28.3.431

Knutsson A, Boggild, H. 2010. Gastrointestional disorders among shift workers. Scandinavian Journal of Work, Environment and Health 36:85-95.

Krogius-Kurikka L, Lura, A., Malinen, E., Aarnikunnas, J., Tuimala, J., Paulin, L., Makivuokko, H., Kajander, K., Palva, A. 2009. Microbial community analysis reveals high level phylogentetic alterations in the overall gastrointestional microbiota of diarrhoeapredominant irritable bowel sufferers. BMC Gastroenterology 9:95. 10.1186/1471230X-9-95

Ley RE, Turnbaugh, P.J., Klein, S., Gordon, J.I. 2006. Microbial ecology: Human gut microboes associated with obesity. Nature 444:1022-1023.

Liu L, Xiao, Q-f., Zhang, Y-l., Yao, S-k. 2014. A cross-sectional study of irritable bowel syndrome in nurses in China: Prevalence and associated psychological and lifestyle factors. Journal of Zheijang University Science B 15:590-597. 10.1631/jzus.B1300159

Liu Y, Zhang, L., Wang, X., Zhang, J., Jiang, R., Wang, X., Wang, K., Liu, Z., Xia, Z., Xu, Z., Nie, Y., Lv, X., Wu, X., Zhu, H., Duan, L. 2016. Similar fecal microbiota signatures in patients with diarrhea-predominant irritable bowel syndrome and patients with depression. Clinical Gastroenterology and Hepatology 14:1602-1611.e1605.

Liu Z, Wei, Z-Y, Chen, J., Chen, K., Mao, X., Liu, Q., Sun, Y., Zhang, Z., Zhang, Y., Dan, Z., Tang, J., Qin, L., Chen, J-H., Liu, X. 2020. Acute Sleep-Wake Cycle Shifts Results in Community Alteration of Human Gut Microbiome. mSphere 5:e00914-00919.

Lowen A, Moreno, C., Holmback, U., Nannernas, M., Tucker, P. 2010. Eating and shift workeffects on habits, metabolism and performance. Scandinavian Journal of Work, Environment and Health 36:150-162.

Lozupone CA, Hamady, M., Kelley, S.T., Knight, R. 2007. Quanitative and qualitative beta diversity measures lead to different insights into factors that structure microbial communities. Applied and Enviornmental Microbiology 73:1576-1585.

Peer) reviewing PDF | (2021:02:57966:1:1:NEW 12 Apr 2021) 
486

487

488

489

490

491

492

493

494

495

496

497

498

499

500

501

502

503

504

505

506

507

508

509

510

511

512

513

514

515

516

517

518

519

520

521

522

523

524

525

526

527

528

529

Lozupone CA, Hamady, M., Knight, R. 2006. UniGrac--an online tool for comparing microbial community diversity in a phylogenetic context. BMC Bioinformatics 7:371-378.

Lozupone CAK, R. 2005. UniFrac: a new phylogenetic method for comparing microbial communities. Applied and Enviornmental Microbiology 71:8228-8235.

McMurdie PJ, Holmes, S. 2013. Phyloseq: an R package for reproducible interactive analysis and graphics of microbiome census data. PLOS One 8:e61217. https://doi.org/10.1371/journal.pone.0061217

Methé BA, Nelson, K.E., Pop, M., Creasy, H.H., Giglio, M.G., Huttenhower, C, Gevers, D., et al. 2012. A framework for human microbiome research. Nature 486:215-221.

Mortas H, Bilici, S., Karakan, T. 2020. The circadian disruption of night work alters gut microbiota consistent with elevated risk for future metabolic and gastrointestinal pathology. Chronobiology International 37:1067-1081. 10.1080/07420528.2020.1778717

Myers JA, Haney, M.F., Griffiths, R.F., Pierse, N.F., Powell, D.M.C. 2015. Fatigue in air medical clinicians undertaking high-acuity patient transports. Prehospital Emergency Care 19:36-43.

Nojkov B, Rubenstein JH, Chey WD, and Hoogerwerf WA. 2010. The Impact of Rotating Shift Work on the Prevalence of Irritable Bowel Syndrome in Nurses. American Journal of Gastroenterology 105:842-847.

Nojkov B, Rubenstein, J.H., Chey, W.D., Hoogerwerf, W.A. 2010. The impact of rotating shift work on the prevalence of irritable bowel syndrome in nurses. American Journal of Gastroenterology 105:842-847.

Pan A, Schernhammer, E.S., Sun, Q., Hu, F.B. 2011. Rotating night shift work and risk of type 2 diabetes: Two prospective cohort studies in women. PLOS Med.

Pittayanon R, Lau, J.T., Yuan, Y., Leontiadis, G.I., Tse, F., Surette, M., Moayyedi, P. 2019. Gut microbiota in patient with irritable bowel syndrome-a systematic review. Gastroenterology 157:97-108.

Pozuelo M, Panda, S., Santiago, A., Mendez., S., Accarino, A., Santos, J., Guarner, F., Azpiroz, F., Manichanh, C. 2015. Reduction of butyrate- and methane-producing microorganisms in patients with irritable bowel syndrome. Scientific Reports 5:12693.

Rajaratnam SMW, Barger, L.K., Lockley, S.W., Shea, S.A., Wang, W., Landrigan, C.P., O'Brien, C.S., Qadri, S., Sullivan, J.P., Cade, B.E., Epstein, L.J., White, D.P., Czeisler, C.A. 2011. Sleep disorders, health and safety in police officers. Journal of the American Medical Association 306:2567-2578.

Rangel I, Sundin, J., Fuentes, S., Repsilber, D., de Vos, W.M., Brummer, R.J. 2015. The relationship between faecal-associated and mucosal-associated microbiata in irritable bowel syndrome patients and healthy subjects. Alimentary Pharmacology and Theraputics 42:1211-1221.

Rigsbee L, Agans, R., Shankar, V., Kenche, H., Khamis, H.J., Michail, S.K., Paliy, O. 2012. Quantitative profiling of gut microbiota of children with diarrhea-predominant irritable bowel syndrome. . American Journal of Gastroenterology 107:1740-1751.

Saberi HR, Moravveji, A.R. 2010. Gastrointestional complaints in shift working and dayworking nurses in Iran. Journal of Circadian Rhythms 8:1-4. 
530

531

532

533

534

535

536

537

538

539

540

541

542

543

544

545

546

547

548

549

550

551

552

553

554

555

556

557

558

559

560

561

562

563

564

565

566

567

568

569

570

571

572

573

574

Salonen A, de Vos, W.M., Palva, A. 2010. Gastrointestinal microbiota in irritable bowel syndrome: Present state and perspectives. Microbiology 156:3205-3215. 10.1099/mic.0.043257-0

Sperber AD, Dumitrascu, D., Fukudo, S., Gerson, C., Ghoshal, U.C., Gwee,K.A., Pali, A., Hungin, S., Kang, J-Y., Min-hu, C., Schmulson, M., Bolotin, A., Friger, M., Whitehead, W. 2017. the global prevalence of IBS in adults remains elusive due to the hetrogeneity of studies: A Rome Foundation working team literature review. Gut Pathology 66:1075-1082.

Suwazono Y, Dochi, M., Sakata, K., Okubo, Y., Oishi, M., Tanaka, K., Kobayashi, E., Kido, T., Nogawa, K. 2008. A longitudinal study on the effect of shift work on weight gain in male Japanese workers. Obesity 16:1887-1893.

Tap J, Derrien, M., Törnblom, H., Brazeilles, R., Cools-Porter, S., Doré, J., Störsrud, S., Le Nevé, B., Öhman, L., Simrén, M. 2017. Identification of an Intestinal Microbiota Signature Associated with Severity of Irritable Bowel Syndrome. Gastroenterology 152:111123. 10.1053/j.gastro.2016.09.049

Thaiss CA, Zeevi, D., Levy, M., Zilberman-Schapira, G., Suez, J.,Tengeter, A.C., Abramson, L., Katz, M.N., Korem, T., Zmora, N., Kuperman, Y., Biton, I., Gilad, S., Harmelin, A., Shapiro, H., Halpern, A., Segal, E., Elinav, E. 2014. Transkingdom Control of Microbiota Diurnal Oscillations Promotes Metabolic Homeostatis. Cell 159:514-529.

Turnbaugh PJ, Hamady, M., Yatsunenko, T., Cantarel, B.,L., Duncan, A., Ley, R.E., Sogin, M.L., Jones, W.J., Roe, B.A., Affourtit, J.P., Egholm, M., Henrissat, B., Health, A.C., Knight, R., Gordon, J.I. 2009. A core gut micrombiome in obese and lean twins. Nature 457:480484.

United States Department of Labor Bureau of Labor Statistics. 2005. Workers on flexible and shift schedules in May 2004. Available at http://www.bls.gov/news.release/flex.toc.htm (accessed July 26 2015).

Voigt RM, Forsyth, C.B., Green, S.J., Mutlu, E., Engen, P., Vitaterna, M.H., Turek, F.W., Keshavarizian, A. 2014. Circadian disorganization alters intestional microbiota. PLOS One 9:1-17.

Vyas MV, Garg, A.X., Iansavichus, A.V., Costella, H., Donner, A., Laugsand, L.E., Janszky, I., Mrkobrada, M., Parraga, G., Hackam, D.G. 2012. Shift work and vascular events: Systematic review and meta-analyssis. British Medical Journal 345:1-11.

Wang H, Garrity, G.M., Tiedje, J.M., Cole, J.R. 2007. Naive Bayesian classified for rapid assignment of rRNA sequences into the new bacterial taxonomy. Applied and Enviornmental Microbiology 73:5261-5270.

Wells MM, Roth, L., Chande, N. 2012. Sleep disruption secondary to overnight call shifts is associated with irritable bowel syndrome in residents: A cross sectional study. The American Journal of Gastroenterology 107:1151-1156.

Youn YH, Kim, H.C., Lim, H.C., Park, J.J., Kim, J-H., Park, H. 2016. Long-term clinical course of post-infectious irritable bowel syndrome after shifellosis: a 10-year follow up study. Journal of Neurogastroenterology\& Motility 22:490-496. 10.5056/jnm15157

Zhen LW, Ann, G.K., Yu, H.K. 2006. Functional bowel disorders in rotating shift nurses may be related to sleep disturbances. European Journal of Gastroenterology \& Hepatology 18:623-627.

PeerJ reviewing PDF | (2021:02:57966:1:1:NEW 12 Apr 2021) 
576

577 


\section{Table 1 (on next page)}

A Comparison of Day and Night Shift Participants 
1 Table 1 Sample Description

2

\begin{tabular}{lccc}
\hline & $\begin{array}{c}\text { Day } \\
\text { Shift } \\
(\mathrm{n}=24)\end{array}$ & $\begin{array}{c}\text { Night Shift } \\
(\mathrm{n}=27)\end{array}$ & P Value \\
\hline Age (mean) & 32.4 & 33.3 & $0.73^{*}$ \\
BMI (mean) & 27.1 & 26.3 & $0.60^{*}$ \\
BMI & 1 & 3 & $0.41^{* *}$ \\
$<20$ & 10 & 8 & \\
$20-24.9$ & 5 & 10 & \\
$25-29.9$ & 8 & 6 & \\
$>30$ & 17 & 16 & $0.56^{* *}$ \\
IBS (Rome III criteria) & & 11 & \\
No & 7 & & \\
Yes & 1 & 2 & $1.0^{* *}$ \\
IBS Symptoms & 1 & 1 & \\
$\quad$ IBS with diarrhea & 5 & 7 & \\
IBS with constipation & & 1 & \\
IBS mixed type & 5 & & \\
IBS un-subtyped & 0 & & \\
*Welsh two-sample t-test & & & \\
** Fisher's exact test & & &
\end{tabular}


Figure 1

Principal Components Analysis Plot by ID

(A) Bray-Curtis

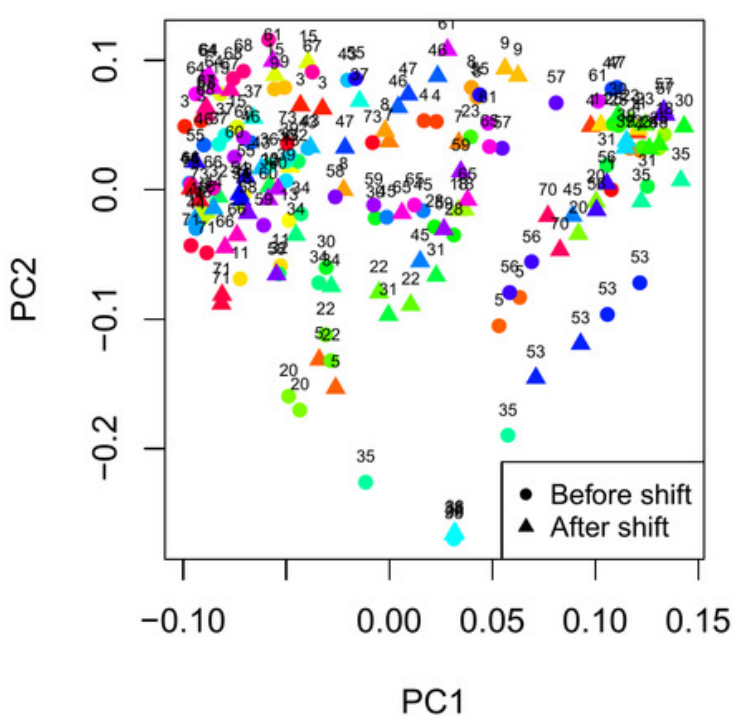

(B) Jaccard

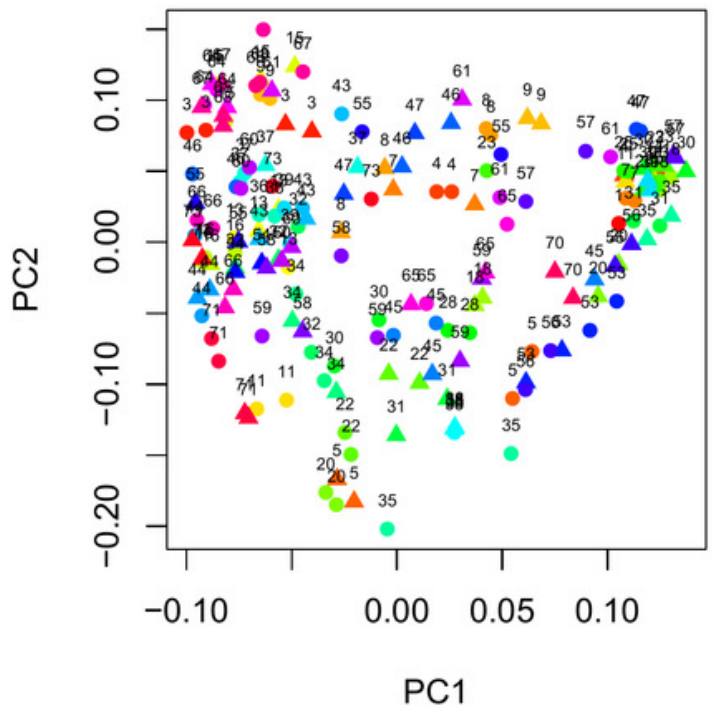


Figure 2

\section{Alpha Diversity by Shift Type}

(A) $\log$ Chao1

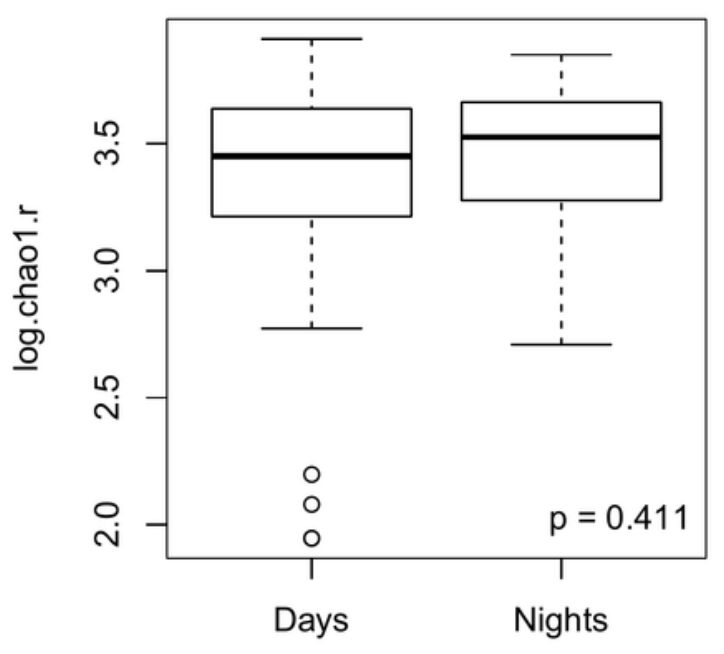

(B) Shannon

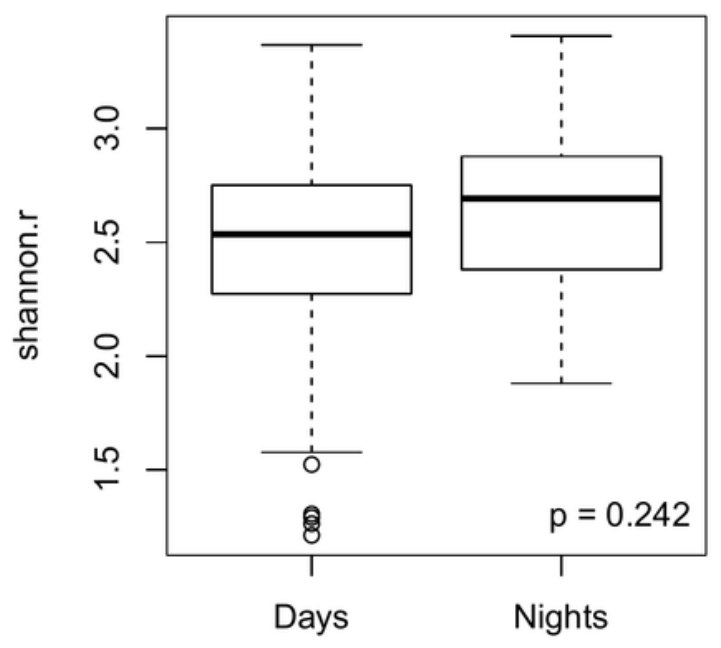


Figure 3

PCoA Plot Comparing Beta Diversity by Shift Type

(A) Bray-Curtis

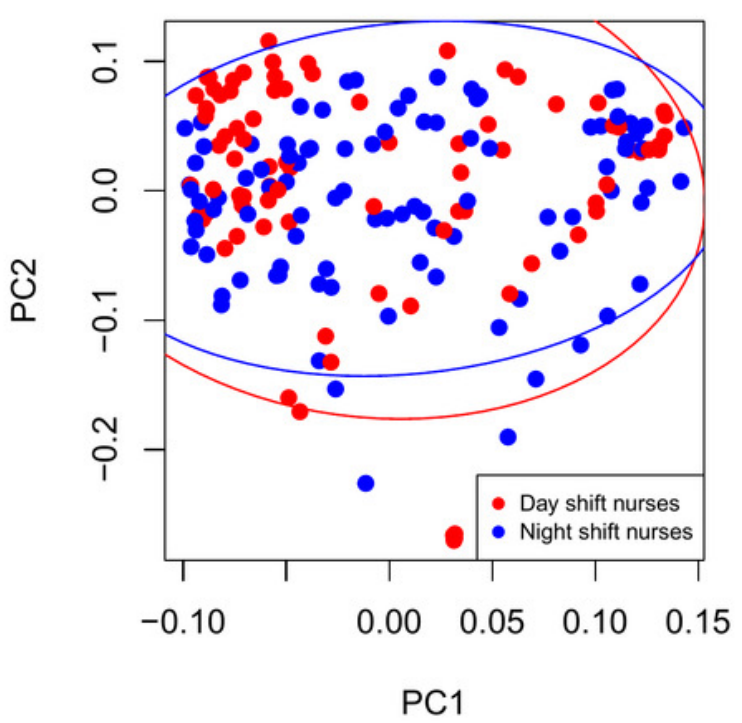

(B) Jaccard

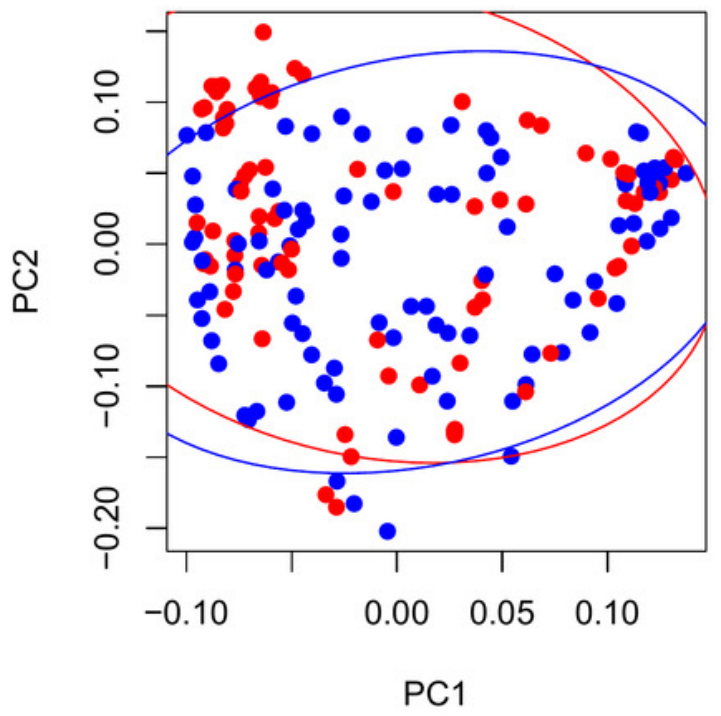


Figure 4

Changes in Alpha Diversity from the Beginning to the End of the Shift by Shift Type

(A) $\log$ Chao1

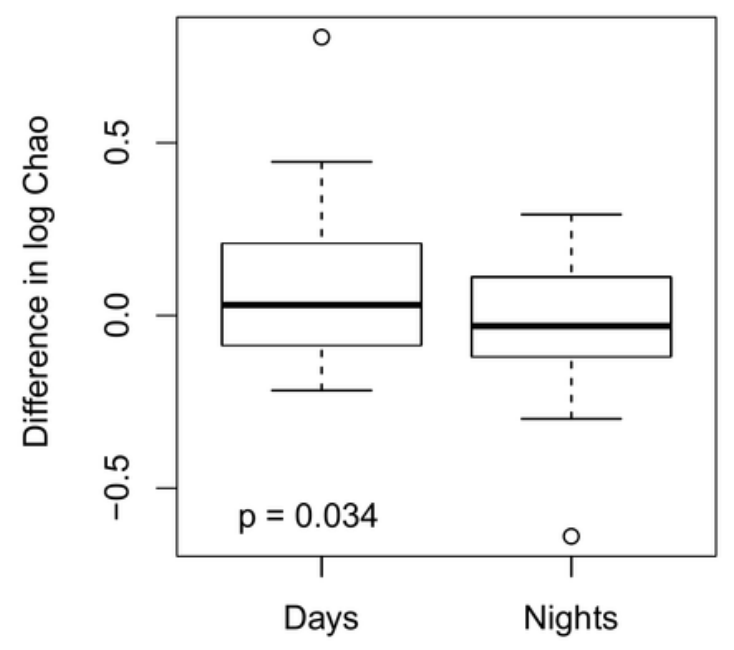

(B) Shannon

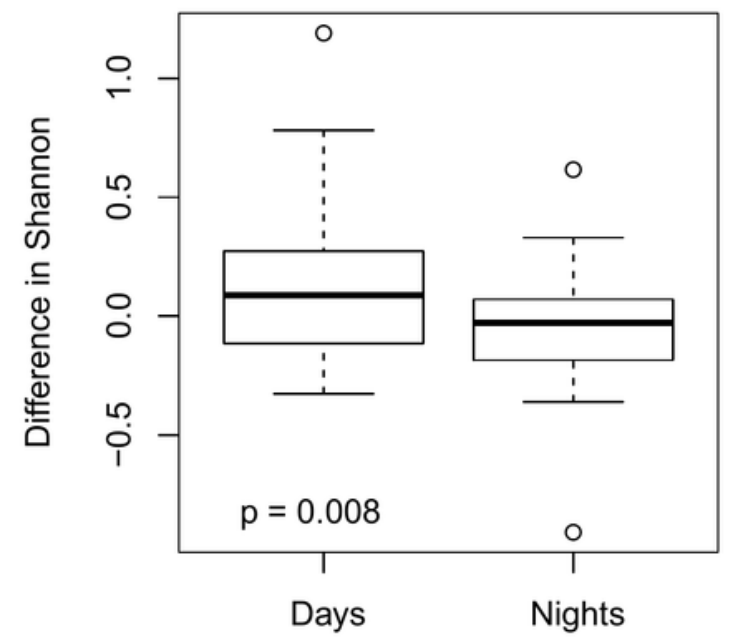


Figure 5

Alpha Diversity by Presence of Absence of IBS

(A) $\log$ Chao1

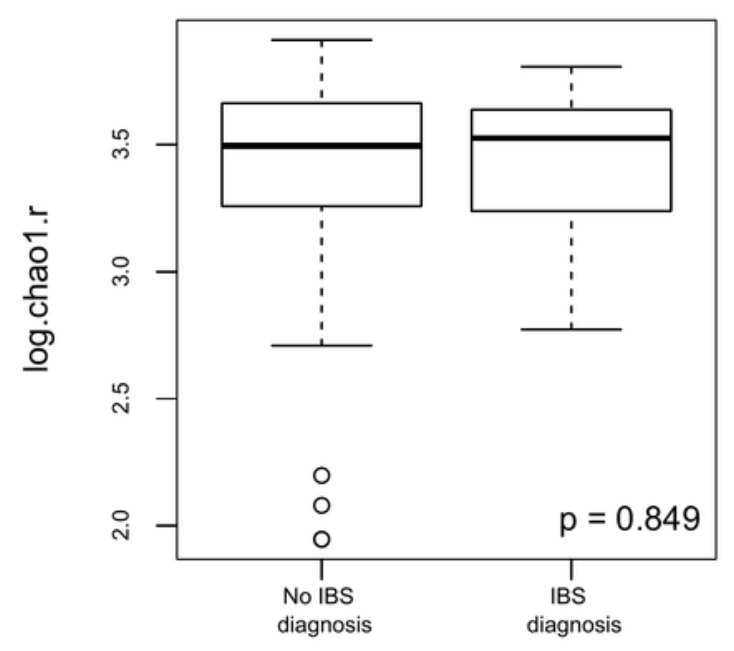

(B) Shannon

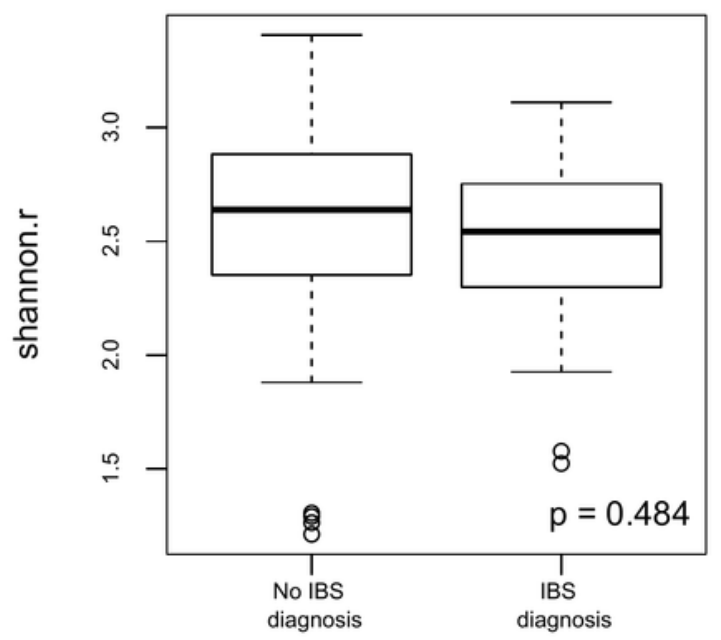

